\title{
Characterization of Epitaxial LSMO Films Grown on STO Substrates
}

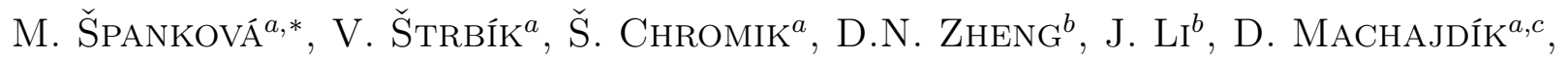 \\ A.P. KobZeV ${ }^{c}$, T. PleceniK ${ }^{d}$ AND M. SoJKOVÁ ${ }^{a}$ \\ ${ }^{a}$ Institute of Electrical Engineering SAS, Dúbravská cesta 9, 84104 Bratislava, Slovakia \\ ${ }^{b}$ Beijing National Laboratory for Condensed Matter Physics and Institute of Physics, Chinese Academy of Sciences, \\ Beijing 100190, China \\ ${ }^{c}$ Joint Institute for Nuclear Research, Dubna, Moscow Region, Russia \\ ${ }^{d}$ Faculty of Mathematics, Physics and Informatics, Comenius University, \\ Mlynská Dolina F1, 84248 Bratislava, Slovakia
}

\begin{abstract}
Epitaxial manganite $\mathrm{La}_{0.67} \mathrm{Sr}_{0.33} \mathrm{MnO}_{3}$ (LSMO) layers, with a thickness of $20-50 \mathrm{~nm}$, are prepared on single crystal (001) $\mathrm{SrTiO}_{3}$ (STO) substrates by pulsed laser deposition technique. Structural characterization (composition analysis, surface morphology), investigated by the Rutherford backscattering spectroscopy and atomic force microscopy, reveals the growth of stoichiometric LSMO films with a smooth surface (root-mean-square value of $0.21-1.6 \mathrm{~nm})$. The prepared LSMO films possess high Curie temperature $(\approx 412 \mathrm{~K})$, low room temperature resistivity $(1-2 \mathrm{~m} \Omega \mathrm{cm})$ and maximum of temperature coefficient of resistivity TCR $=2.7 \% \mathrm{~K}^{-1}$ at $321 \mathrm{~K}$.
\end{abstract}

DOI: $10.12693 /$ APhysPolA.131.848

PACS/topics: 68.37.Ps, 75.47.Lx, 75.70.Ak, 81.15.Fg

\section{Introduction}

Perovskite manganites with general formula $\mathrm{A}_{1-x} \mathrm{~B}_{x} \mathrm{MnO}_{3}$ (where $\mathrm{A}$ is a lanthanum (La) and $\mathrm{B}$ an alkaline-earth (Ba, $\mathrm{Ca}, \mathrm{Sr}$ ) element) have attracted much attention due to the observation of a colossal magnetoresistance (CMR) effect [1, 2]. The CMR effect occurs in the $0.2>x>0.5$ doping range. Among these materials $\mathrm{La}_{1-x} \mathrm{Sr}_{x} \mathrm{MnO}_{3}$ group $\left(\mathrm{La}_{0.7} \mathrm{Sr}_{0.3} \mathrm{MnO}_{3}\right.$, $\mathrm{La}_{0.67} \mathrm{Sr}_{0.33} \mathrm{MnO}_{3}$ ) has been studied intensively because of their high Curie temperature $\left(T_{\mathrm{C} \text {,bulk }} \approx 370 \mathrm{~K}\right)$. Manganite thin films have many potential applications exploiting their magnetic properties, e.g. low-cost magnetic sensors and high-density memory applications [3].

In this work we investigate structural, magnetic, and electrical properties of epitaxial $\mathrm{La}_{0.67} \mathrm{Sr}_{0.33} \mathrm{MnO}_{3}$ (LSMO) thin films deposited by pulsed laser deposition. The films were grown on single crystalline substrates (001) $\mathrm{SrTiO}_{3}$ (STO). The quality of the film was proved by structural as well as physical properties characterization.

\section{Experimental}

The bulk manganite LSMO can be described as a slightly deformed pseudocubic perovskite lattice with a lattice parameter $a_{\text {bulk }}=0.3876 \mathrm{~nm}$ and a unit cell angle $90.26^{\circ}$. The STO substrate has a cubic structure with a lattice parameter of $0.3905 \mathrm{~nm}$. The lattice misfit between the bulk LSMO and the STO substrate is $0.75 \%$, inducing biaxial tensile strain in the LSMO films.

\footnotetext{
*corresponding author; e-mail: marianna.spankova@savba.sk
}

The LSMO films were deposited onto a one-side polished (001) oriented single crystalline STO substrates using pulsed laser deposition (PLD) system (MBE/PLD-2000). A $\mathrm{KrF}$ excimer laser operating at $248 \mathrm{~nm}$ with a pulse width of $20 \mathrm{~ns}$, repetition rate of $10 \mathrm{~Hz}$ and energy density of $6 \mathrm{~J} / \mathrm{cm}^{2}$ was used to grow the LSMO films. The temperature of the substrate holder was kept to $850^{\circ} \mathrm{C}$ and the oxygen pressure was set to $53 \mathrm{~Pa}$ during the deposition. After the deposition, the films were cooled down at a rate of $20^{\circ} \mathrm{C} / \mathrm{min}$ in $\mathrm{O}^{2}\left(4 \times 10^{4} \mathrm{~Pa}\right)$. The thickness of the LSMO films were deduced from the growth rate of the films $(6.5 \mathrm{~nm} / \mathrm{min})$ and X-ray reflectivity measurements. It was varied between 20 and $50 \mathrm{~nm}$.

The Rutherford backscattering spectrometry (RBS) measurements were performed on Van de Graaff accelerator using $3.055 \mathrm{MeV} \mathrm{He}^{2+}$ ions with an angle between the sample surface and the beam $30^{\circ}$. The backscattered particles were detected at $170^{\circ}$ with respect to the incident beam.

The surface morphology of the LSMO films was examined by atomic force microscopy (AFM) carried out on NT-MDT NTegra Aura in semicontact mode.

The temperature dependence of the resistivity was measured using a standard dc four-probe technique in a temperature range of 4-480 K. The magnetic properties of the films were measured at temperatures ranging from 10 to $500 \mathrm{~K}$, using a SQUID magnetometer (MPMS-XL7) and a vibrating sample magnetometer (PPMS-9T), all from Quantum Design. The magnetization vs. temperature $(M(T))$ measurements were performed in the field of $5 \mathrm{mT}$ applied in the film plane. 


\section{Results and discussion}

The prepared LSMO films exhibited very good crystal quality. X-ray diffraction analyses confirmed that our LSMO grew epitaxially on the STO substrates [4].

The LSMO films exhibit a homogeneous distribution of all presented elements in the film through the whole film thickness. The calculated composition of the examined LSMO film obtained from the RBS measurement is $\mathrm{La}_{0.65} \mathrm{Sr}_{0.35} \mathrm{Mn}_{1.04} \mathrm{O}_{2.96}$, i.e. the average cation composition ratio La:Sr:Mn of the film corresponds to the target composition.

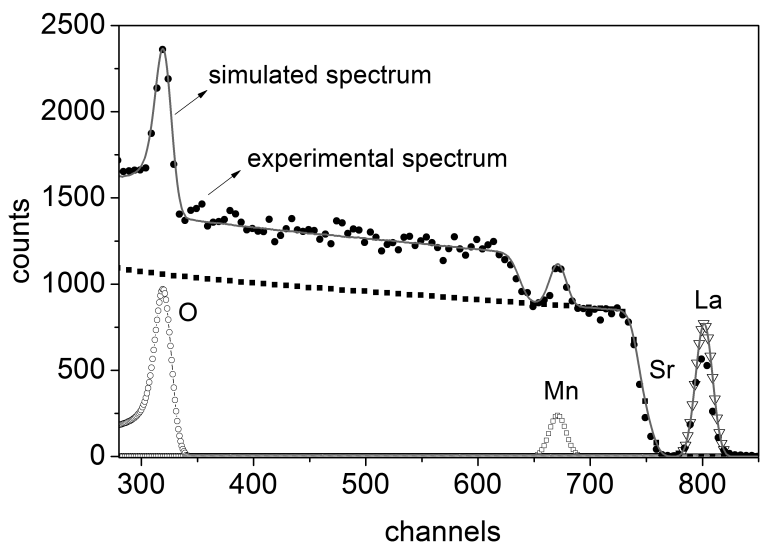

Fig. 1. RBS spectrum of a $30 \mathrm{~nm}$ thick LSMO grown on STO. The collected RBS data were analysed using BS software.

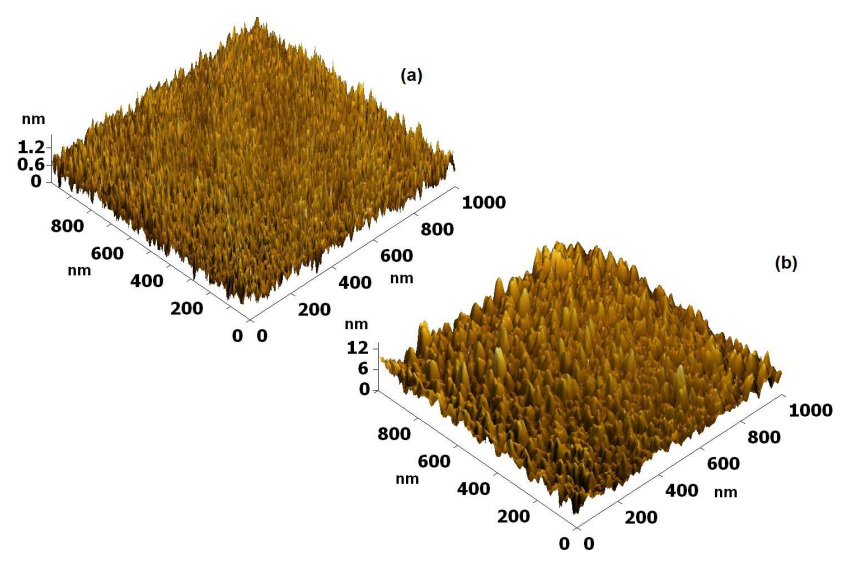

Fig. 2. AFM surface image of a $20 \mathrm{~nm}$ (a) and a $50 \mathrm{~nm}$ (b) thick LSMO film. The surface consists of uniformly distributed crystallites with a dimension of $20 \mathrm{~nm}$ and $40 \mathrm{~nm}$, respectively.

The morphology of the film surface, analysed by AFM, is generally smooth for the LSMO films of different thickness. In the case of a $20 \mathrm{~nm}$ thick LSMO film the root-mean-square (RMS) value is $0.21 \mathrm{~nm}$ for the $1 \times 1 \mu \mathrm{m}^{2}$ area. The surface is composed of grains with a mean diameter size of $20 \mathrm{~nm}$ (Fig. 2). The surface

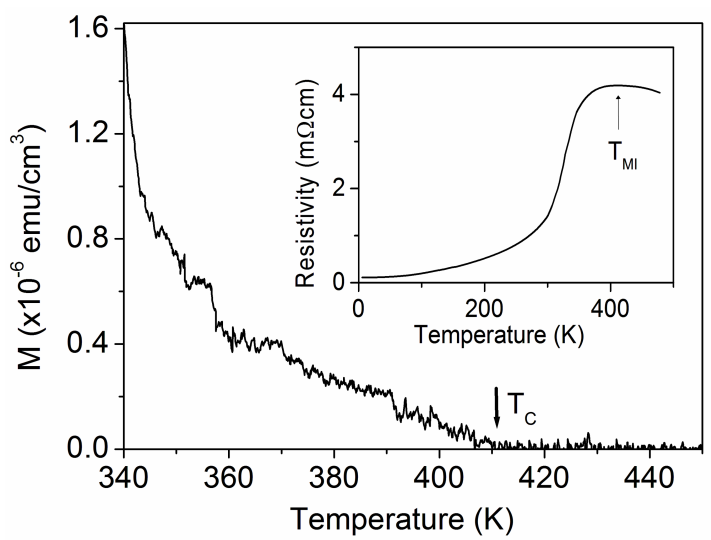

Fig. 3. Temperature dependence of magnetization of a $20 \mathrm{~nm}$ thick LSMO film grown on STO substrate. $T_{\mathrm{C}}$ indicates the transition PM to FM state. Inset shows the resistivity vs. temperature dependence of this film with maximum at temperature $T_{m i}$.

roughness and the size of the crystallites increases with the film thickness - for a $50 \mathrm{~nm}$ thick LSMO the RMS value of $1.6 \mathrm{~nm}$ and the grain size of about $40 \mathrm{~nm}$ were detected.

STO with a small lattice misfit to the LSMO (0.75\%) is a suitable substrate for the preparation of LSMO thin films with high Curie temperature $\left(T_{\mathrm{C}}\right)$ and low resistivity $\rho$ as it is shown in Fig. 3 . The $T_{\mathrm{C}}$ of the LSMO film was determined from the temperature dependence of magnetization at $412 \mathrm{~K}$ as the temperature of paramagnetic $(\mathrm{PM})(M=0) /$ ferromagnetic $(\mathrm{FM})(M>0)$ transition. The temperature of resistivity maximum $T_{m i}$ (corresponding to metal-insulator transition) (Fig. 3, inset) was estimated at temperature $T_{m i}=412 \mathrm{~K}$. The $T_{\mathrm{C}}$ and $T_{m i}$ values coincide according to double-exchange theory explaining the ferromagnetic properties of manganites. The typical room temperature resistivity is about 1-2 $\mathrm{m} \Omega \mathrm{cm}$ and maximal resistivity changes were observed at the temperatures around $330 \mathrm{~K}$. The maximum of temperature coefficient of resistivity $\mathrm{TCR}=2.7 \% \mathrm{~K}^{-1}$ was achieved at $321 \mathrm{~K}$. Moreover, Méchin et al. [5] found that the level of $1 / f$ noise at room temperature decreases with increased value of $T_{m i}$, therefore the presented LSMO thin film with $T_{m i}=412 \mathrm{~K}$ seems to be a good candidate for room temperature application as sensor.

In our previous works $[6,7]$ we presented the polynomial fit expression $\rho(T)=\rho_{0}+a_{2} T^{2}+a_{N}\left(T-T_{0}\right)^{4.5}$, well describing the electrical and magnetotransport properties of the LSMO film in a wide range of temperature $(2-380 \mathrm{~K})$ and magnetic field $0-9 \mathrm{~T}$. In this paper we demonstrate the approximation of $\rho(B)$ dependences taken at various temperatures (Fig. 4 , inset). At first, the $\rho(T, B)$ dependences were measured and approximated (Fig. 4, main part). From these approximations we can determine the magnetic field dependences of fitting parameters $\rho_{0}(B), a_{2}(B), a_{N}(B), T_{0}(B)$ [7] and then subsequently approximate the $\rho(B)$ dependences at a given 


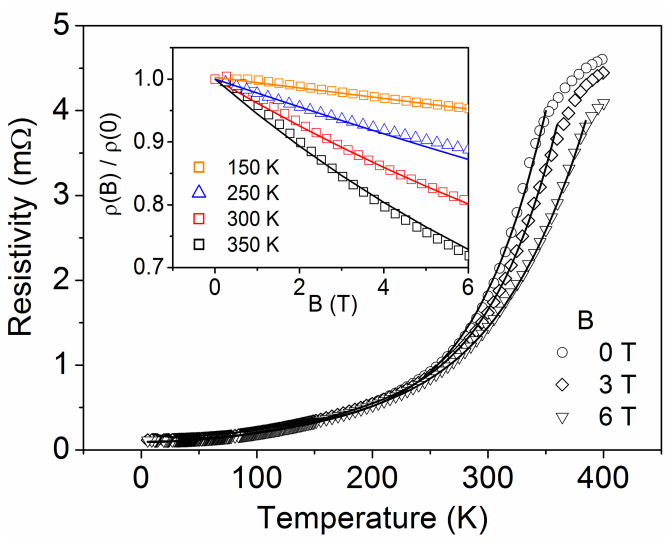

Fig. 4. Main part: temperature dependences of resistivity of a $20 \mathrm{~nm}$ thick LSMO film in magnetic field 0 , 3 , and $6 \mathrm{~T}$ (points) and approximations (full lines) with polynomial expression. Inset: the dependences of resistivity on applied magnetic field at various temperatures (points) and approximations (full lines) using polynomial fit expression with magnetic field dependent fitting parameters.

temperature. The reason for approximation of electrical and magnetic properties of the LSMO is to analyze individually each component of the total LSMO resistivity $\rho$.

\section{Conclusions}

Structural, electrical and magnetotransport properties of LSMO films grown on STO substrates have been studied. We presented the possibility to approximate the dependences of the resistivity on applied magnetic field in temperature range of $150-350 \mathrm{~K}$ where the largest magnetoresistivity of the films was observed. The epitaxial LSMO films exhibited significantly higher temperature of metal-insulator transition $T_{m i}$, as well as the Curie temperature than it was presented for the bulk LSMO. Since high $T_{m i}$ corresponds to low noise, the investigated LSMO films are considered to be a good candidate in room temperature sensor applications.

\section{Acknowledgments}

This work was supported by the Slovak Research and Development Agency, APVV-0494-11, APVV-14-0613, Slovak Grant Agency for Science, VEGA 2/0120/14, by Structural Funds of the European Union by means of the Agency of the Ministry of Education, Science, Research and Sport of the Slovak republic in the project "CENTE II" ITMS code 26240120019. The authors acknowledge the fruitful collaboration within COST Action MP1308 TO-BE.

\section{References}

[1] S. Jin, T.H. Tiefel, M. McCormack, R.A. Fastnacht, R. Ramesh, L.H. Chen, Science 264, 413 (1994).

[2] M.-J. Casanove, C. Roucau, P. Baules, J. Majimel, J.-C. Ousset, D. Magnoux, J.F. Bobo, Appl. Surf. Sci. 188, 19 (2002).

[3] C. Moreno, C. Munuera, S. Valencia, F. Kronast, X. Obradors, C. Ocal, Nano Lett. 10, 3828 (2010).

[4] M. Španková, V. Štrbík, E. Dobročka, Š. Chromik, M. Sojková, D.N. Zheng, J. Li, Vacuum 126, 24 (2016).

[5] L. Méchin, S. Wu, B. Guillet, P. Perna, C. Fur, S. Lebargy, C. Adamo, D.G. Schlom, J.M. Routoure, 0.1088/0022-3727/46/20/202001J. Phys. D Appl. Phys. 46, 202001 (2013).

[6] V. Štrbík, Š. Chromik, J. Eletric. Eng. 63, 270 (2012).

[7] V. Štrbík, M. Reiffers, Š. Chromik, M. Španková, Acta Phys. Pol. A 126, 212 (2014). 